\title{
Study of Moisture Absorption by Ranitidine Hydrochloride: Effect of \% RH, Excipients, Dosage Forms and Packing Materials
}

\author{
S.M. Ashraful Islam ${ }^{1}$, Md. Amir Hossain ${ }^{1}$, A. N.M. Hamidul Kabir ${ }^{2}$, \\ Shaila Kabir ${ }^{3}$ and Md. Khalid Hossain ${ }^{3}$
}

\author{
${ }^{1}$ Department of Pharmacy, The University of Asia Pacific, Dhaka-1209, Bangladesh \\ ${ }^{2}$ Department of Applied Chemistry \& Chemical Technology, University of Dhaka, Dhaka-1000, Bangladesh \\ ${ }^{3}$ Department of Pharmaceutical Chemistry, Faculty of Pharmacy, University of Dhaka, Dhaka-1000, Bangladesh
}

\begin{abstract}
Moisture absorption by ranitidine at three different level of relative humidity (RH) has been studied. Percentage of moisture absorption at $22 \%, 57 \%$ and $75 \%$ RH was $0.6 \%, 3.1 \%$, and $4.9 \%$ respectively. Ranitidine blended with various excipients (maize starch, lactose, avicel PH-101, mannitol, povidone K-30, magnesium stearate and purified talc) were also exposed to $75 \%$ relative humidity (\% RH) to observe their effects on moisture absorption. Moisture absorption by ranitidine was either increased or decreased when ranitidine was mixed with various diluents, binders and lubricants. Ranitidine mixed with lactose, aerosil-200 and avicel PH-101 absorbed minimum amount of moisture while ranitidine mixed with maize starch absorbed maximum. On the other hand, ranitidine mixed with mannitol was found to absorb intermediate amount of moisture. Ranitidine mixed with povidone K-30 absorbed almost double moisture than pure ranitidine. Ranitidine mixed with purified talc and magnesium stearate absorbed almost same amount of moisture. The type of dosage form and type of packing materials also influenced moisture absorption. Uncoated tablets absorbed maximum amount of moisture (4.5\%) while coated tablets absorbed minimum (2\%). On the other hand, capsule was found to absorb intermediate amount of moisture (3.4\%). PVC-Alu foil packed uncoated ranitidine tablet absorbed more moisture (3.3\%) than that of Alu-Alu packed uncoated ranitidine tablet $(0.16 \%)$.
\end{abstract}

Key words: \% RH, ranitidine hydrochloride, moisture, absorption

\section{INTRODUCTION}

Moisture plays remarkable negative role in pharmaceutical product, particularly for solid dosage forms. Both physical and chemical stability of some drugs are affected by moisture. Moisture is absorbed on the surface of solid drugs and increases the rate of decomposition, causes agglomeration and dissolution of drugs. ${ }^{1}$

Correspondence to: Md. Khalid Hossain

Tel: 880-2-9661920-79, Ex-144; Fax: 880-2-8612069

E-mail: hossainkhalid2004@yahoo.com

Dhaka Univ. J. Pharm. Sci. 7(1): 59-64, 2008 (June)
Presence of moisture possesses a critical challenge on drug stability. Moisture accelerates the hydrolysis of drug as well as facilitates reaction with other excipients, thereby affecting stability and shelf life of the final product. The effect of moisture absorption on the shelf life of coated tablets has been reported previously. ${ }^{2}$ Generally, the initial moisture level as well as the inherent tendency of the active ingredients and excipients to uptake water from the surrounding environment governs the moisture absorption pattern of the final product. The hygroscopic nature of excipients and active ingredients should be considered in designing the formulation. Water sorption or desorption by drugs 
and excipients is not always reversible and absorbed moisture may not be easily removed during drying which directly affects the drug stability.

Moisture can affect the way in which a system accepts aqueous granulating solutions. The moisture content and rate of moisture uptake are the functions of temperature and humidity. Moisture affects the tableting characteristics in granulation process. Hygroscopicity data can aid in the design of tablet manufacturing areas. Moisture sensitive drugs should not be combined with hygroscopic excipients. Packing material should be chosen to suit the product as moisture directly affects the drug stability.

The physical properties and tablet performances are also influenced by moisture absorption pattern. The effect of moisture absorption on disintegration time has been reported earlier. ${ }^{3}$ The rate and extent of moisture absorption influence the selection of packaging materials. The relationship between moisture absorption with dissolution profile in different packing condition was recorded previously. ${ }^{4}$

Ranitidine is extensively used as anti-ulcerant. For a hygroscopic drug ranitidine, which absorbs moisture from the environment, its physical and chemical instability in the presence of moisture has a major impact on the choice of formulation excipients, the selection of processing method, and the design of the product package. ${ }^{5,6}$ Investigators have described moisture uptake rate of various commercial brands of ranitidine tablet in Bangladesh and inter-brand variability in the rate and extent of moisture absorption. ${ }^{7}$ The aim of the present study was to investigate the effect of \% relative humidity, excipients (especially diluents, binders, lubricants), various dosage forms and packing materials on moisture absorption of ranitidine hydrochloride.

\section{MATERIALS AND METHODS}

Ranitidine hydrochloride (USP), maize starch, lactose, mannitol, avicel PH-101, aerosil-200, povidone K-30, talc, and magnesium stearate were obtained as generous gift from Hudson Pharmaceuticals Limited.
Moisture uptake study by ranitidine at different relative humidity. A controlled humidity environment was produced using laboratory desiccators containing saturated solutions of various salts. $^{8}$ Potassium acetate, sodium bromide, and sodium chloride were used $^{5}$ to provide a RH of 22, 57 and $75 \%$, respectively at $25^{\circ} \mathrm{C}$. Percentage of $\mathrm{RH}$ was measured by a hygrometer.

One gm ranitidine hydrochloride (USP) was taken in a glass petridish and spreaded uniformly in the petridish in a thin layer. The petridish was then placed in the $75 \% \mathrm{RH}$ chamber to observe the moisture uptake. The weight increase due to moisture absorption was measured by taking weight at different intervals such as intervals of 10, 20, 30, 60, 90, 120, 150 and $180 \mathrm{~min}$ by an electronic balance (Metler, Switzerland).

In the same way, one gm ranitidine hydrochloride was placed in each 57\% RH and 22\% $\mathrm{RH}$ chamber and amount of moisture absorbed determined.

Moisture uptake study of drug-diluent mixture. Maize starch, lactose, mannitol, avicel PH-101 and aerosil-200 were taken as diluents. One gm of each diluent was mixed with one gm of ranitidine hydrochloride in a glass container by rotating the container vigorously for 20 minutes and the mixtures were assigned as M1, M2, M3, M4, and M5, respectively.

The mixtures M1, M2, M3, M4, and M5 were taken in five different glass containers and spreaded uniformly in the containers in a thin layer. The containers were then placed in the constant humidity (75\% RH) chamber. The weight increase due to moisture absorption was measured by taking weight at different intervals such as $10,20,30,60,90,120$, 150 and $180 \mathrm{~min}$. Electronic balance (Metler, Switzerland) was used to record the weight.

Moisture uptake study of drug-binder mixture. Povidone K-30 was taken as binder. One gm ranitidine hydrochloride and one gm povidone $\mathrm{K}-30$ were taken in a V-type glass container and 
mixed thoroughly by rotating the container for 20 minutes. The mixture was assigned as B1.

The mixture B1 was taken in a glass container and spreaded uniformly in a thin layer. The container was then placed in the constant humidity $(75 \% \mathrm{RH})$ chamber. The weight increase due to moisture absorption was measured by taking weight at different intervals such as 10, 20, 30, 60, 90, 120, 150 and $180 \mathrm{~min}$. Electronic balance (Metler, Switzerland) was used to record the weight.

Moisture uptake study of drug-lubricant mixture. Purified talc and magnesium stearate were used as lubricants. One gm of each lubricant was mixed thoroughly with $1 \mathrm{gm}$ ranitidine hydrochloride powder in V-type glass containers. Two mixtures L1 (ranitidine + purified talc) and L2 (ranitidine + magnesium stearate) were thus prepared.

The mixtures L1, and L2 were taken in two different glass containers and spreaded uniformly in a thin layer. The containers were then placed in the constant humidity (75\% RH) chamber. The weight increase due to moisture absorption was measured by taking weight at different intervals such as 10, 20, 30, 60, 90, 120, 150 and $180 \mathrm{~min}$.

Moisture uptake study of various dosage forms of ranitidine. At first, $83.75 \mathrm{gm}$ ranitidine hydrochloride was mixed with 37.50 gm avicel PH-101 and 37.50 gm maize starch. Then $10 \mathrm{ml}$ of $50 \%$ povidone K-30 solution in ethanol was added and mixed thoroughly. The mass was dried with continuous shaking in a tray dryer at $40^{\circ} \mathrm{C}$ for 30 minutes. The mass was passed through the sieve no \# 10 to produce uniform granules. Then 3.75 gm magnesium stearate and 3.75 gm purified talc were mixed with the granules. The granules were then compressed in $10 \mathrm{~mm}$ dies and punches. Each tablet contained $150 \mathrm{mg}$ ranitidine.

Film coating of tablet. Tablets were coated by spraying hydroxy propyl methyl cellulose (HPMC) dispersion in ethanol (96\%) and methylene chloride mixture. The tablets were taken in a small rotary pan and heated. The pan was rotated at $20 \mathrm{rpm}$. Inlet and outlet temperatures were 80 and $60^{\circ} \mathrm{C}$, respectively.
Preparation of capsule. Capsules were prepared by using the following formula:

$\begin{array}{ll}\text { Ranitidine hydrochloride } & 83.75 \mathrm{gm} \\ \text { Aerosil-200 } & 2.50 \mathrm{gm} \\ \text { Magnesium stearate } & 5.00 \mathrm{gm}\end{array}$

All the materials were mixed in a glass container and encapsulated manually in empty hard gelatin capsule (EHGC) \# 2. Each capsule contained $150 \mathrm{mg}$ ranitidine.

Uncoated tablets, coated tablets and capsules were taken in three different glass containers and spreaded uniformly in the containers. The containers were then placed in the constant humidity ( $75 \% \mathrm{RH})$ chamber. The weight increase due to moisture absorption was measured by taking weight at different intervals such as $1,2,3,5,6,7,20,32,44$, 56, 68 and 92 hrs.

Moisture uptake study of PVC-Alu foil and Alu-Alu foil packed uncoated tablets. Uncoated tablets were packed in two ways: one in PVC-Alu foil packing and another in Alu-Alu foil packing. All the packing operations were done in Hudson Pharmaceuticals Limited by using automatic blister packing machine. Room temperature and \% $\mathrm{RH}$ were $25^{\circ} \mathrm{C}$ and $60 \%$, respectively.

The packed tablets were taken in two different glass containers. The containers were then placed in the constant humidity $(75 \% \mathrm{RH})$ chamber. The weight increase due to moisture absorption was measured by taking weight at different intervals e.g., $1,7,14,21,30,60$ and 90 days.

\section{RESULTS AND DISCUSSION}

Effect of relative humidity on the moisture uptake by ranitidine. The moisture absorption study of ranitidine was conducted in different controlled environment. The weight gain at different time interval was taken as moisture gain. Figure 1 shows moisture uptake profiles for 1 gm ranitidine hydrochloride at three different level of relative humidity such as 75,57 and $22 \%$. The amount of moisture uptake was seen to be higher for samples exposed to a higher relative humidity for the three- 
hour time period. The figure indicates that ranitidine exposed to higher relative humidity absorbed maximum amount of moisture. The dependence of moisture uptake on relative humidity has been reported by Li et al. ${ }^{5}$ which is similar to the present study. This is very important for the selection of manufacturing environment for ranitidine tablet.

The rate of moisture absorption was also calculated from the best fit lines of the Figure 1 and presented as bar diagram (Figure 2). It has been observed that ranitidine exposed to higher relative humidity showed high rate of moisture absorption whereas the slowest rate was found with ranitidine exposed to lower relative humidity. Form this experiment, it is clear that the rate and extent of moisture absorption by ranitidine depend on relative humidity. So, it is better to select an environment of low relative humidity for the manufacturing of ranitidine tablet.

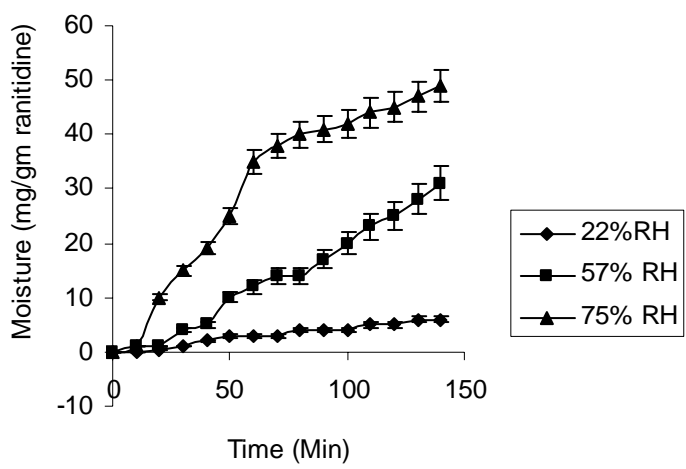

Figure 1. Effect of \% RH on the moisture absorption by ranitidine. Data points are the means and S.D. of triplicate runs



Figure 2. Moisture absorption rate of ranitidine. Data points are the means and S.D. of triplicate runs
It is also noted that all three moisture uptake curves exhibited biphasic characteristics: an initial linear portion followed by a curvilinear segment. This biphasic moisture uptake profile can be further explained with respect to the moisture uptake process. The surface of the particles absorbs moisture rapidly and saturated solution was formed in the adsorbed layer. Then moisture penetrates into the particles slowly and as a result moisture absorption rate decreased forming a biphasic moisture absorption curve.

Effect of diluents on the moisture uptake by ranitidine. Figure 3 shows moisture uptake profiles for pure ranitidine and ranitidine mixed with various diluents (lactose, maize starch, avicel PH-101, mannitol, aerosil-200) at $75 \%$ relative humidity and $25^{\circ} \mathrm{C}$ temperature. The figure indicates inter-diluents variability in rate and extent of moisture absorption

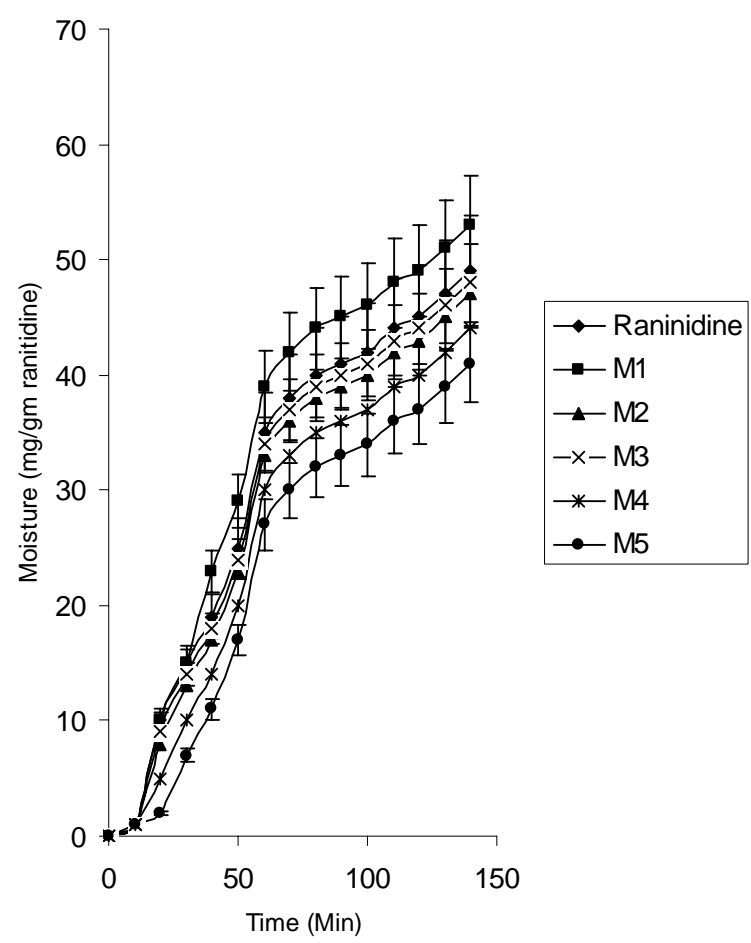

Figure 3. Effect of diluents on the moisture absorption by ranitidine. Data points are the means and S.D. of triplicate runs

by ranitidine. Ranitidine mixed with lactose, aerosil200 and avicel PH-101 absorbed minimum amount of moisture while ranitidine mixed with maize starch absorbed maximum. On the other hand, ranitidine 
mixed with mannitol was found to absorb intermediate amount of moisture. The result is very important in selecting diluents for tableting of ranitidine. Avicel PH-101 and aerosil-200 can be used with ranitidine as filler. Lactose absorbs minimum amount of moisture but it can discolor amine drug like ranitidine in the presence of metallic stearate. ${ }^{9-10}$

Effect of binder on the moisture uptake by ranitidine. The extent of moisture absorption by pure ranitidine and ranitidine mixed with binder (povidone $\mathrm{K}-30$ ) is shown in Figure 4. This figure indicates that ranitidine mixed with povidone K-30 absorbed almost double moisture than that of pure ranitidine. This is because povidone K-30, itself is a hygroscopic material. So, it is better to avoid povidone K-30 in ranitidine tablet if other suitable alternative is available or minimum amount of povidone K-30 should be used in ranitidine tablet formulation.

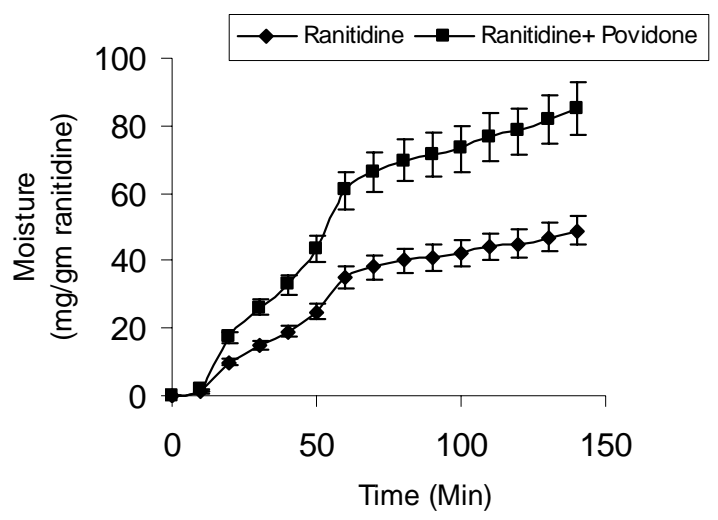

Figure 4. Effect of povidone K-30 on the moisture absorption by ranitidine. Data points are the means and S.D. of triplicate runs

Effect of lubricants on the moisture uptake by ranitidine. The extent of moisture absorption by pure ranitidine and ranitidine mixed with purified talc and magnesium stearate is shown in Figure 5. This figure indicates that ranitidine mixed with lubricants absorbed almost same amount of moisture as pure ranitidine. So lubricant has little effect on the moisture absorption by ranitidine.

Effect of dosage forms on the moisture uptake by ranitidine. The extent of moisture absorption by pure ranitidine, ranitidine capsule, ranitidine coated tablet and ranitidine uncoated tablet is shown in Figure 6. This figure indicates that pure ranitidine absorbed maximum amount of moisture while different dosage forms of ranitidine absorbed less



Figure 5. Effect of lubricants on the moisture absorption by ranitidine. Data points are the means and S.D. of triplicate runs

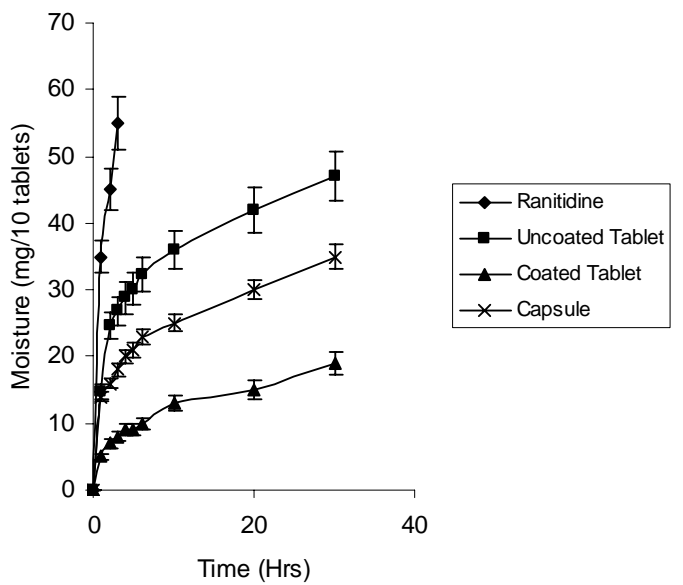

Figure 6. Effect of dosage forms on the moisture absorption by ranitidine. Data points are the means and S.D. of triplicate runs

amount of moisture although inter dosage form variability in moisture absorption is clear. Among the dosage forms uncoated tablet absorbed maximum amount of moisture while coated tablet absorbed minimum. On the other hand, capsule was found to 
absorb intermediate amount of moisture. Uncoated tablets melted gradually while the coated tablets became soft.

Pure ranitidine absorbed higher amount of moisture than tablet. This is because tablet is a compressed dosage form. Moist air cannot penetrate easily in the tablet core. On the other hand, coated tablet absorbed less moisture than uncoated tablet. Capsule absorbed an intermediate amount of moisture. This is because gelatin cannot prevent the moisture penetration.

This study indicates that ranitidine tablet must be marketed as coated form; uncoated tablet is not suitable for commercial supply. On the other hand, ranitidine can be supplied as capsule dosage form as it absorbs less moisture than uncoated tablet. But before that, interaction of ranitidine with gelatin and other component of empty hard gelatin shell should be studied.

Effect of packing materials on the moisture uptake by ranitidine. The extent of moisture absorption by PVC-Alu foil packed uncoated ranitidine tablet and Alu-Alu foil packed uncoated ranitidine tablet was shown in Figure 7. The figure indicates that PVC-Alu foil packed uncoated



Figure 7. Effect of packing materials on moisture absorption by ranitidine. Data points are the means and S.D. of triplicate runs

ranitidine tablet absorbed more moisture than AluAlu packed uncoated ranitidine tablet. The PVC-Alu foil packed tablet became brownish and soft after 2 months. On the other hand, Alu-Alu packed ranitidine tablet absorbed a little moisture and no discoloration was found in the tablet after 2 months.
So, it is better to pack the ranitidine tablet in Alu-Alu blister packing.

\section{CONCLUSION}

Different data generated in this experiment indicates that \% $\mathrm{RH}$ of manufacturing environment has a great effect on the moisture level of ranitidine tablets as ranitidine absorbs maximum amount of moisture at higher \% RH. This higher moisture level is responsible for various physical stability problems of ranitidine tablets. Therefore, by controlling the $\%$ RH of manufacturing environment, we can overcome the various stability problems of ranitidine tablet manufacturing. $20-25 \% \mathrm{RH}$ is considered as the optimum moisture level for ranitidine tablet processing. Excipients, dosage forms and packing materials have also effect on the moisture absorption by ranitidine. So, a judicious combination of excipients and technical selection of packing materials can reduce the rate and extent of moisture absorption.

\section{REFERENCES}

1. Bently's Text Book of Pharmaceutics, 8th Edition 1984. (Rawlins, E.A. (ed.) Bailliere Tindall, UK. pp. 147-148.

2. Nakabayashi, K., Shimamoto, T. and Mima, H. 1980. Stability of packaged solid dosage form. II. Shelf life prediction for packaged sugar coated tablets liable to moisture and heat damage. Chem. Pharm. Bull. 28, 10991106.

3. Hasegawa, A., Nakagawa, H. and Sugimoto, L. 1984. The mechanism of disintegration time increase of tablets containing hydroxylproprylcellulose by moisture absorption. Yakugaku Zasshi. 104, 544-547.

4. Savile 1995. Relationship between moisture absorption with dissolution profile. Int. J. Pharm. 224, 39-49.

5. Luk Chi, Li et al. 1994. A study of the moisture-uptake kinetics of a hygroscopic pharmaceutical powder.

6. British Pharmacopoeia 2002. Properties of ranitidine hydrochloride.. 1, 1481-1482.

7. Kohinur B, Mohiuddin A.Q. and Rudmila F. 2002. Study of moisture absorption rate and morphological changes of ranitdine tablets. Bangladesh Pharm. J. 12, 23-26.

8. Martes, L. 1963. In: Handbook of Analytical Chemistry (eds) Mcgraw Hill, New York, pp 329.

9. Castello, R. and Mattocks, A. 1962. J. Pharm. Sci. 51, 106.

10. Duvall, R. N. et al. 1965. J. Pharm. Sci. 54, 607. 
